\title{
The Influence of Ground Contact and Visible Horizon on Perception of Distance and Size under Severely Degraded Vision
}

\author{
Kristina M. Rand, Margaret R. Tarampi, Sarah H. Creem-Regehr, and William B. Thompson \\ University of Utah
}

\begin{abstract}
For low vision navigation, misperceiving the locations of hazards can have serious consequences. Potential sources of such misperceptions are hazards that are not visually associated with the ground plane, thus depriving the viewer of important perspective cues for egocentric distance. In Experiment 1, we assessed absolute distance and size judgments to targets on stands under degraded vision conditions. Normally sighted observers wore blur goggles that severely reduced acuity and contrast, and viewed targets placed on either detectable or undetectable stands. Participants in the detectable stand condition demonstrated accurate distance judgments, whereas participants in the undetectable stand condition overestimated target distances. Similarly, the perceived size of targets in the undetectable stand condition was judged to be significantly larger than those in the detectable stand condition, suggesting a perceptual coupling of size and distance in conditions of degraded vision. In Experiment 2, we investigated size and implied distance perception of targets elevated above a visible horizon for individuals in an induced state of degraded vision. When participants' size judgments are inserted into the size-distance invariance hypothesis (SDIH) formula, distance to above-horizon objects increased compared to those below the horizon. Together, our results emphasize the importance of salient visible ground-contact information for accurate distance perception. The absence of this ground-contact information could be the source of perceptual errors leading to potential hazards for low vision individuals with severely degraded acuity and contrast sensitivity.
\end{abstract}

\section{Keywords}

Low vision; navigation; distance perception; size perception; visual accessibility

\begin{abstract}
Everyday navigation throughout ones environment may seem like a trivial task due to years of experience, but in fact it involves a complex interaction between visual, cognitive, and motor systems. We must select from a vast array of visual stimuli those items that will optimize our movement through space. Despite this complexity, it has been suggested that explicit effort may not be required to perform simple navigation goals (Fajen \& Warren, 2003). Instead, information is updated continually as a result of visually-streamed information. To date, much is still not known about which visual cues are utilized when perceiving the absolute scale of spaces beyond distances of about $2 \mathrm{~m}$. Such information about scale is critical to path planning and navigation. This knowledge becomes even more relevant for individuals trying to navigate with low vision, as fewer cues are available to inform scale of space. The current study explores judgments of objects located off the ground plane by normally sighted individuals experiencing simulated severely degraded acuity and contrast sensitivity. Our findings suggest that when visual information was
\end{abstract}

Please address correspondence to: Kristina M. Rand, Department of Psychology, University of Utah, 380 S. 1530 E., Rm 502, Salt Lake City, UT 84112, kristina.rand@utah.edu. 
available to connect the off-ground target to the ground plane, individuals were able to make accurate judgments of the absolute distance to a target location. However, when groundcontact information is not clearly visible, systematic errors in distance and size judgments occur.

Navigation through environments is generally successful for both normal and low vision populations despite the potential complexities. Successful navigation is often accomplished by individuals with low vision in the absence of external aids (Ludt \& Goodrich, 2002). The primary focus of existing low vision navigation research is obstacle avoidance while walking, which is an important component to mobility (Kuyk, Elliott, Biehl, \& Fuhr, 1996; Long, Rieser, \& Hill, 1990; Lovie-Kitchin, Soong, Hassan, \& Woods, 2010; Marron \& Bailey, 1982; Pelli, 1987; Turano, Broman, Bandeen-Roche, Munoz, Rubin, West, \& SEE project team, 2004). This research however has not directly addressed the ability to recover absolute distance and the general scale of the space. Being able to recover absolute distance and spatial scale of an environment is critical to navigation. Recently, the accurate perception of absolute distance that has been found experimentally in normal vision (e.g. Loomis, Da Silva, Fujita, \& Fukusima 1992; Philbeck \& Loomis, 1997; Rieser, Ashmead, Talor, \& Youngquist, 1990; Thomson, 1983) has been replicated in severely degraded viewing conditions (Tarampi, Creem-Regehr, \& Thompson, 2010). While these experiments provide important baseline information about absolute distance perception with severely degraded vision, judgments were made in simple environments to single targets located on the ground plane. Although the objects we interact with on a daily basis often make contact with the ground plane, sometimes the point of ground contact is ambiguous or hard to see. Real world examples of objects that may not make visual contact with the ground include furniture with small or thin legs, low material contrast between stands and the surrounding ground surface, and lights located in bollards (see Figure 1 for one real-world example). Our current study examined absolute distance judgments to elevated targets while varying the amount of available information to indicate that targets were not on a ground plane or directly supported by some structure in contact with the ground plane.

The ground plane has been shown to play a large role in both relative and absolute distance perception. According to Gibson's ground theory, use of the ground plane is not surprising given that many important environmental structures that we encounter make direct or indirect contact with this surface (Gibson, 1950). Gibson suggested that in the absence of cues to suggest that a target is off the ground, judged distance is consistent with the assumption that the target is on the ground plane. An illustration of this effect can be found in Figure 2. Findings by Meng and Sedgwick $(2001 ; 2002)$ support ground theory by suggesting that participants will use ground plane information, when available, when making relative distance judgments. This was found even when the target was not in direct contact with the ground surface but the relationship to the ground plane could be extracted through mediating surfaces. Also demonstrating the relevance of the ground plane in distance perception, a target's position relative to a visible horizon has been found to influence relative distance information (Ozkan \& Braunstein, 2010). Taken together, this and related work suggest that the ground plane plays a significant role in distance perception; viewers likely default to assuming that objects make contact with the ground plane unless otherwise there is evidence to the contrary. When visible information suggests that targets are off the ground, viewers still appear to use the ground plane in distance judgments.

One mechanism thought to aid in absolute distance perception is the angle of declination from a line-of-sight parallel to the ground plane to a line-of-sight to the target location (Sedgwick, 1983). The horizon-distance relation is one of few cues that might allow a viewer to recover absolute distance information to objects located more than three meters 
from the viewer's location on the ground plane. Ooi, Wu, and $\mathrm{He}$ (2001) found support for this theory: when individuals viewed an environment through prism goggles, effectively lowering perceived eye level and decreasing the angle of declination, absolute distance estimates were overestimated. Further, it has been suggested that visible horizon information serves as a frame of reference for the horizon-distance relation under degraded viewing conditions (Rand, Tarampi, Creem-Regehr, \& Thompson, 2011). This is the case even in indoor environments when the ground plane is truncated, and therefore is not a close approximation of a true horizon. The current study expands on this finding by exploring the role of the visible horizon as an implicit indicator of an object's off-ground location.

The current study also explores the relationship between size and distance in profoundly degraded visual conditions. Previous studies have used size estimates as an indicator of distance judgments for several reasons, including methodological challenges and to avoid possible cognitive biases (Gogel, 1984; Loomis \& Philbeck, 2008). The possibility of using size as an indirect measure of distance follows from the size-distance invariance hypothesis (SDIH), which asserts that the ratio of perceived size to perceived distance is equal to the ratio of actual size to actual distance, perhaps scaled by a multiplicative constant (Gilinsky, 1951; Kilpatrick \& Ittelson, 1953). This relationship between perceived size and distance can be expressed in a form that can predict perceived distance given perceived size and the actual size and distance:

$$
D^{\prime}=\left(\frac{1}{C}\right)\left(\frac{D}{S}\right) S^{\prime}
$$

where $D^{\prime}$ is apparent distance, $S^{\prime}$ is apparent size, $D$ and $S$ are actual distance and size, and $C$ is an observer constant (Gogel, Loomis, Newman, \& Sharkey, 1985).

While many studies have found coupling between perceived size and distance (Gilinsky, 1951; Gogel, 1969; Kilpatrick \& Ittelson, 1953), there are also multiple studies suggesting that the percepts are independent (Epstein, Park, \& Casey, 1961; Haber \& Levin, 2001; Mon-Williams \& Tresilian, 1999; Ono, Muter, \& Mitson, 1974). Epstein et al. (1961) suggested that there is no a priori reason why distance and size should be dependent on one another, and data from three experiments failed to meet the criteria of the size-distance invariance hypothesis. To our knowledge, no one has tested the relationship between size and distance percepts under severely reduced contrast and acuity. In the current study, we were interested in whether size-distance invariance would emerge under severely degraded viewing conditions.

Although there is great variability in the causes and manifestations of profound low vision ${ }^{1}$, it is often associated with a substantial reduction in acuity and contrast sensitivity. The current study explores the effect of visible ground contact information on the ability to determine the distance to objects under such conditions. This is done in two experiments in which normally-sighted individuals perform tasks that involve distance judgments while wearing blur goggles that severely degrade acuity and contrast. While our ultimate goal is to explore this issue in a low vision patient population, the initial use of normally sighted participants coupled with artificially reduced acuity and contrast aided in carefully controlling our experiments. We further restricted viewing to only the dominant eye of each participant for several reasons. In many cases, the reductions in acuity and contrast

\footnotetext{
${ }^{1}$ There is a lack of standardization in terminology categorizing different levels of low vision. In this paper, we use the term profound low vision to mean a visual acuity in the range from 20/500 to 20/1000, as defined in the Visual Standards Report prepared for the International Council of Ophthalmology, Sydney, Australia (2002). It is important to note that while low vision is usually categorized in terms of acuity, reductions in acuity are only one of the visual deficits associated with low vision.
} 
sensitivity associated with profound low vision are accompanied by degradations in stereo vision. The restriction to monocular viewing also allows us to concentrate on the visual information arising from ground contact cues, while avoiding potential experimental issues that may arise from confounding this information with that coming from binocular stereo.

These experiments advance our understanding of severely degraded viewing conditions by addressing three main questions. First, how does perception of off-ground targets change in the absence of visual information conveying the elevated position of the target? In Experiment 1, distance judgments made to objects on visible stands were compared to judgments made to objects on stands that were of sufficiently low contrast relative to their backgrounds that they could not be seen through the blur goggles. This allowed us to assess whether visual information directly connecting the target to the ground is sufficient for accurate distance perception under low vision conditions. Second, do size and distance percepts behave as predicted by the size-distance invariance hypothesis under severely degraded viewing conditions? To explore this question, size judgments were also measured in Experiment 1 and compared to distance judgments. Third, how are size, and the implied distance of an object, perceived with severely degraded contrast sensitivity and acuity when targets are elevated above a visible horizon? This issue is important because the direction of the line-of-sight to targets above the horizon does not provide any direct information about the location of those targets relative to the ground plane. Experiment 2 explored this question by assessing judgments of targets that were elevated both below and above the visible horizon were assessed.

\section{Experiment 1}

The first experiment used both blind walking and size judgments as direct and indirect measures of distance judgments, respectively, to targets elevated off the ground plane. The blind walking task involves the participant walking without vision to a previously viewed target without feedback about their position or the position of the stimuli. The distance walked is used as an indication of the perceived egocentric distance to the target. As demonstrated through decades of research, blind walking is an established method of accurately obtaining distance judgments to targets up to $20 \mathrm{~m}$ (e.g. Fukusima, Loomis, \& Da Silva, 1997; Loomis et al., 1992; Loomis \& Philbeck, 2008; Philbeck \& Loomis, 1997; Rieser et al., 1990; Thomson, 1983). The blind walking procedure was used to test for differences between targets placed on detectible versus undetectable stands when observed in severely degraded viewing conditions. It was predicted that objects elevated with undetectable stands would be judged as farther away than targets elevated with detectable stands. This finding would be consistent with Gibson's (1950) ground theory that suggests that unless contradicted by other evidence indicating its position, the human visual system will treat objects as though they are located on the ground plane. Also of interest in Experiment 1 was the relationship between size and distance judgments for individuals in severely degraded viewing conditions.

\section{Method}

Participants-A total of 22 individuals ( 7 males, 15 females, mean age 22.27 years) participated in Experiment 1. Participants were compensated with either partial course credit or were paid ten dollars. Four participants were removed from analyses. Two were removed due to poor vision because it was important that baseline vision was normal or corrected-tonormal prior to the low vision manipulation. Additionally, one was removed because of confusion regarding instructions, and one due to reported dizziness prior to the completion of the experiment. All remaining participants had normal or corrected-to-normal vision. 
Materials-To eliminate the distraction of environmental noises, all participants were equipped with noise-cancelling headphones. Participants listened to background pink noise on an mp3 player through the headphones while the experimenter spoke to the participant above the noise through a microphone. All participants also wore blur goggles that allowed for monocular viewing through their dominant eye. As the experiment was not a parametric study, but designed to test potential differences in perception of objects when the stand was detectable or not detectable, a single level of blur was utilized. The blur goggles were welding goggles with a theatrical lighting gel (ROSCO Cinegel \#3047: Light Velvet Frost), resulting in an average tested acuity of 1.51 (logMAR) or 20/647 (Snellen) and an average tested log contrast sensitivity 0.46 , fitted in the dominant eye of participants in both Experiment 1 and 2. The non-dominant eye was blacked out. The tested field of view through the viewing aperture was an average of 72 degrees in the horizontal, and 68 degrees in the vertical. To block overhead and surrounding light cues, participants also wore a hood made of blackout fabric over the blur goggles when they were not viewing the stimuli. Participants viewed three white, Styrofoam spheres with diameters of $20.32 \mathrm{~cm}, 30.48 \mathrm{~cm}$, and $40.64 \mathrm{~cm}$. The spheres were placed on one of two cylindrical stands. The stands were both $45.72 \mathrm{~cm}$ tall cardboard cylinders with diameter of $8.11 \mathrm{~cm}$. The stand used for the undetectable stand condition was painted matte gray, while the stand for the detectable condition was painted matte black. This resulted in low and high contrast, respectively, with the gray carpet in the experiment classroom (See Figure 3 for an illustration of the conditions). In addition, an assessment of detectability was conducted with 6 participants confirming that the detectable stand was visible and the undetectable stand was not visible when viewed through the blur goggles (see Appendix). All participants were tested in a classroom with approximately $7.7 \mathrm{~m}$ x $10.1 \mathrm{~m}$ of walkable space. Participants' fixed starting point on all trials was positioned $9.83 \mathrm{~m}$ from the back wall of the classroom. This starting position ensured that for all participants and all target distances used in the experiment, the entire target was below the line-of-sight to the visible horizon.

Design-The experiment used a 2 (condition) $\times 2$ (task order) $\times 3$ (distance) $\times 3$ (target size) design, with between-subjects variables of stand condition (undetectable stand, detectable stand) and task order (distance first, size first), and within-subjects variables of target distance $(1.5 \mathrm{~m}, 2.7 \mathrm{~m}, 4 \mathrm{~m})$ and target size $(20.32 \mathrm{~cm}, 30.48 \mathrm{~cm}, 40.64 \mathrm{~cm})$.

Procedure-Participants were first asked to sign the consent form, followed by a measurement of their eye heights. They were then tested for eye dominance using the holein-the-card test (Durand \& Gould, 1910), and administered two vision tests. Each participant was tested first with the blur goggles and second with normal monocular vision on the ETDRS Acuity and Pelli-Robson Contrast Sensitivity tests. Next, participants read the experiment instructions, and were trained on the task procedures in a hallway outside of the classroom. As task order was counterbalanced, the initial training phase depended on the order of the conditions (distance first or size first). Participants in the walk-first condition were first familiarized with walking without vision. This involved a multi-step procedure where the participant was first guided by the experimenter, followed by independent blind walking with stop-and-start instructions from an experimenter. Next, participants were given two practice trials of blind walking to a previously viewed target without feedback. They were then outfitted with the noise-cancelling headphones, $\mathrm{mp} 3$ player, microphone receiver, blur goggles, and hood. Then they were guided into the classroom without prior vision.

Once the participant was led to the starting location, 22 blind walking trials were administered. Two trials were practice trials with no feedback, 2 trials were dummy trials at 2 and 3.5 meters, and 18 were randomized experimental trials. The dummy trials were used in order to reduce the chance that participants would be aware of repeated distances. For the 18 experimental trials, participants walked twice to each of three targets at each critical 
distance $(1.5,2.7$ and $4 \mathrm{~m})$. On each trial, participants were asked to lift the hood and open their dominant eye to view the target. They were informed that the targets on which to base their judgments were white objects of varying size in the space in front of them. After 5 seconds of viewing, participants were instructed to lower the hood. They were then instructed to walk toward the perceived target location and to stop when they believed they were standing at the center of the white target's location. The second experimenter removed targets and stands in advance. Measurements were taken from the center of their final position, and then participants were guided back to the starting location.

Upon completion of the distance judgment block, participants were led outside of the classroom for instructions regarding the size judgment task. Participants were instructed to hold out their hands so that the gap between them corresponded to the size of object they viewed, as though they were standing right next to the target, reaching to pick it up. The experimenter then demonstrated a correct size judgment made to a distant target to emphasize the importance of taking distance into account. They were then given two practice trials without feedback, and were led back into the classroom wearing the hood, blur goggles, and noise cancelling headphones. Participants were led to the same starting location and performed the same 22 trials as in the distance judgment block, in a different randomized order. On each trial, participants raised the hood to view the target. After viewing the target location for 3 seconds, a viewing restrictor was placed so that it allowed for unrestricted view of the target, but did not allow participants to view their hands. As participants were able to view the object unrestricted prior to the placement of the restrictor, together with the fact that the viewing restrictor reduced the field of view by a negligible amount, it is unlikely that the viewing restrictor influenced perceptions of the environment. They were then asked to make their size judgments. When the participant felt as though their hands were in the correct position, they were asked to say 'okay'. At that point, the experimenter lowered the participant's hood and measured the distance between their hands.

For participants in the size-first condition, the experiment began with the size judgment training and size judgment block prior to the blind walking training and distance judgment block. All other procedures were identical. Upon completion of both experimental blocks, all participants were debriefed.

\section{Results}

Distance judgments were converted into accuracy ratios to equate judgments made across distances and enable collapsing across distances. These were calculated by dividing the walked distance on each trial by the actual target distance. A ratio value of greater than 1.0 indicates that targets were judged to be in a location farther than the actual target location. Participants in the detectable stand condition showed accuracy ratios of .996 ( $\mathrm{SD}=.15), 1.05$ $(\mathrm{SD}=.22)$, and $1.05(\mathrm{SD}=.19)$ to targets placed at $1.5 \mathrm{~m}, 2.7 \mathrm{~m}$, and $4 \mathrm{~m}$, respectively, while accuracy ratios for those in the undetectable stand were 1.64 (SD=.39), 1.67 ( $\mathrm{SD}=.41)$, and $1.63(\mathrm{SD}=.29)$. A 3 (target distance) $\times 2$ (stand condition) $\times 2$ (task order) mixed ANOVA was conducted on the accuracy ratios averaged across the three target sizes with target distance as a within-subject factor and stand condition (detectable/undetectable) and task order (walk first/size first) as between-subjects factors. It was predicted that participants in the undetectable stand condition would overestimate the target distance when compared to the detectable stand condition. There was a main effect of stand condition $[\mathrm{F}(1,14)=22.91$, $\left.\mathrm{p}<.001, \eta^{2}=.621\right]$ in the predicted direction (see Figure 4). The stand condition X target distance interaction, main effect of target size, as well as the main effect of task order, did not reach significance.

Distances for the projected target locations, indicating where the target would appear if the object was assumed to be in direct contact with the ground plane, were calculated using 
participants' individual eye heights. These values averaged $2.1 \mathrm{~m}, 3.8 \mathrm{~m}$, and $5.6 \mathrm{~m}$ to targets placed at $1.5 \mathrm{~m}, 2.7 \mathrm{~m}$, and $4 \mathrm{~m}$, respectively. These values were converted to a ratio representing the walked distance/projected distance. For the undetectable stand condition, ratios were $1.14,1.16$, and 1.14 . A one-sample t-test comparing the ratio values to a value of 1 did not reach significance $(\mathrm{p}=.083)$, suggesting there was no compelling evidence for a difference in walked and projected distance.

Ratio values for size judgments were also calculated by dividing the size judgment by the actual target size. Ratios are shown in Figure 5. A 3 (target distance) $\times 2$ (stand condition) $\times$ 2 (task order) mixed ANOVA was conducted on the size judgment ratios, averaged across the three target sizes, with target distance as a within-subject factor and stand condition and task order as between-subjects factors. Similar to the blind walking results, there was a main effect of stand condition $\left[\mathrm{F}(1,14)=22.75, \mathrm{p}<.001, \eta^{2}=.619\right]$, such that participants in the undetectable stand condition judged targets to be larger than those in the detectable stand condition. The effects of target distance, task order, and order X condition interaction were not significant.

Since Experiment 1 involved judgments of both apparent size and apparent distance, it allowed for the evaluation of the degree to which perceived size can be used as an indirect measure of perceived distance under conditions of severely degraded contrast and acuity. This requires solving for the constant $C$ in Equation 1 and then confirming that Equation 1 is a good model for predicting $D^{\prime}$ (apparent distance) given $S^{\prime}$ (apparent size). The constant $C$ in Equation 1 is normally assumed to be specific to individual viewers. However, since we want to use results from Experiment 1 to interpret size judgments in Experiment 2 as indirect measures of apparent distance and since Experiment 2 involved a different set of participants than did Experiment 1, we solved for a value of $C$ for the population of participants in Experiment 1. This was done using linear regression through the origin to compare the dependent variable $D^{\prime}$ to the independent variable $(D / S) S^{\prime}$, where $D^{\prime}$ is apparent distance, $S^{\prime}$ is apparent size, and $D$ and $S$ are actual distance and size as in Equation 1 . The reason for using regression through the origin rather than ordinary leastsquares regression is that the model relating perceived distance to perceived size shown in Equation 1 has only a multiplicative term, with no additive term. The distances in Equation 1 are along the line-of-sight, while the walked distances in Experiment 1 are along the floor. As a result, prior to the regression analysis all distances along the floor were converted to line-of-sight distances by projecting onto the line-of-sight determined by the target location and height and the participant's eye height.

Figure 6 shows a plot of the mean $(D / S) S^{\prime}$ and the resulting linear regression line of $D^{\prime}$ values (apparent distance as indicated by blind walking), both with respect to the line-ofsight. Nine plotted data points per condition represent each of 3 target sizes by each of 3 target distances, collapsed across participants. As discussed above, regression lines were forced through the zero intercept. The slope of the regression line provides an estimate for the reciprocal of an average scaling constant $C$, which is needed in order to use size judgments as an indirect indicator of perceived distance for Experiment 2. The overall constant value for Experiment 1 was .760, with constants of .815 and .735 for the detectable and undetectable stand conditions individually. In addition, a bivariate correlation was conducted on the observed line-of-sight distance values and distance values predicted by Equation 1 using the calculated $\mathrm{C}$ value. The square of the correlation provides an evaluation of the fit of the regression through origin model (Hocking, 2003). This yielded an overall squared correlation value of .976 for both conditions combined, with .984 for the detectable stand condition and .960 for the undetectable stand condition. 
To explore the between-participant variability in the $\mathrm{C}$ values, we computed the individual $\mathrm{C}$ values for each participant. The average of these values was $.80(\mathrm{SD}=.26)$. For the detectable stand condition, the average $\mathrm{C}$ value was $.84(\mathrm{SD}=.24)$, with an average of .76 $(\mathrm{SD}=.30)$ for the undetectable stand condition.

\section{Discussion}

Three important findings emerged from Experiment 1. First, despite severely degraded viewing conditions, participants who were provided visual information to suggest that targets were located off the ground plane (detectable stand condition) were able to accurately judge absolute distances to targets elevated on stands. Second, when that information was not available (undetectable stand condition), participants overestimated the target distance, suggesting that they were grounding targets to a location similar to where they were visually projected. Size was underestimated for the visible stands condition. Taken together with the near accurate distance judgments in this condition, this is consistent with $C<1$ in the regression analysis. One likely possibility for this underestimation is that the blur goggles influence the perceived location of object boundaries, causing the objects to appear smaller. (Some research with non-degraded vision has shown that size judgments are overestimated with respect to perceived distance (Gogel et al., 1985), though it is difficult to make comparisons with our present results using very different stimuli and methodologies.) Both size and distances were overestimated in the undetectable stands condition. Given the similar values for the constant $C$ in both the visible and undetectable stands conditions, it is plausible to assume that the overestimation of size is due to the overestimation of distance, not some change in the size estimation process itself compared with the visible stand case.

\section{Experiment 2}

Results from Experiment 1 suggest that when explicit information is available connecting a raised target to the ground plane immediately below the target, observers accurately perceive the target location. Experiment 2 extended these results to include the situation where some targets appeared above the visible horizon, without visible support connecting the target to the ground. Under these circumstances, the targets are no longer seen occluding a portion of the ground surface. As such, there is no way that the ground surface can contribute information about target position. Participants viewed targets located either on the ground, or elevated off the ground by a thin cable. Previous work in the same classroom space has demonstrated that alterations to the floor/wall boundary alter distance judgments (Rand et al., 2011). Based on this research, we were confident that participants could perceive the visible horizon created from the floor/wall boundary. In Experiment 2, target positions were varied such that some targets were presented above the visible horizon. Although raising the targets above this boundary was likely to provide information that the target was not located on the ground plane, exactly where targets would be perceived was unknown. Several possibilities seemed plausible: 1.) Consistent with ground contact, with a lack of information suggesting the contrary, objects would be perceived as located on the back wall surface, 2.) Participants would treat the surface above the visible horizon as a continuation of the ground plane, or 3.) Participants would perceive the objects presented above the visible horizon as neither located on the ground plane nor on the back wall surface, but instead, somewhere in front of the back wall. Due to the potential concern of participants judging the above horizon targets to be located on the back wall, we thought it unsafe to use blind walking as a distance measure. Participants in Experiment 2 made size judgments to provide an indirect measure of distance perception as this relationship was established in Experiment 1. 
Participants-A total of 14 new individuals ( 3 males, 11 females, mean age 22.14 years) participated in Experiment 2. Participants were compensated with either partial course credit or were paid ten dollars. Participants had normal or corrected-to-normal vision.

Materials-The same size spheres that were used in Experiment 1 were used in Experiment 2. For the second experiment, the spheres were painted a shade of green that contrasted approximately evenly in reflectivity with the floor and with the back wall. The effects of illumination were such that the contrast between spheres and background varied substantially around the circumference of the sphere and across conditions, but in all cases the boundaries of the spheres were clearly visible, even through the blur goggles. Holes were cut in each sphere to allow for a thin wire to pass through. Eleven sets of 6-foot wires were created to control for where the sphere was projected for participants of varying eyeheight such that a new set was used for every $3 \mathrm{~cm}$ difference in eye-height when targets were placed at $4 \mathrm{~m}$ from the starting location. Each wire was marked with 3 pieces of tape indicating the appropriate location for the bottom of the sphere, and the spheres could be moved up and down the wire until the appropriate height was reached. This control ensured that the target positions located below the visible horizon occluded approximately the same location on the ground surface, and targets positioned above the horizon occluded approximately the same location on the back wall, regardless of participant height. Small weights covered with floor-colored fabric were placed under each of three fixed wire loops at 3, 4, and 5 meters from the participants' starting location. Under the weights, extendable strings were placed to allow tension when a cable was hung from the ceiling (see Figure 7).

Design-The experiment used a 4 (target position) $\times 3$ (target size) design in which target position (ground, below horizon, above horizon, eye-height) and target size (small, medium, large) were within subject variables. The critical target distance was $4 \mathrm{~m}$. Dummy trials at 3 $\mathrm{m}$ and $5 \mathrm{~m}$ were included in the experimental procedure to allow for more randomized trials but were not fully crossed with target size and target position and were not further analyzed.

Procedure-Participants met the experimenter outside of the classroom, signed the consent form, and underwent the same vision test procedure as for Experiment 1.

Participants also wore the same noise cancelling setup, blur goggles, and hood. After reading through written instructions, hearing verbal instructions, and performing two size judgment practice trials, participants were led blindfolded to the starting location in the classroom. Participants performed 38 experimental trials; 2 practice trials, 12 trials at dummy distances ( $3 \mathrm{~m}$ and $5 \mathrm{~m}$ ) with varying target sizes, and 24 randomized critical trials, where each target size was judged twice at each height at the $4 \mathrm{~m}$ distance. Upon completion of the block, participants were guided outside of the classroom for debriefing.

\section{Results}

Size judgments were converted to accuracy ratios by dividing the reported size by the actual target size. A within-subjects ANOVA was conducted on the accuracy ratios as a function of target position for targets at $4 \mathrm{~m}$. There was a main effect of target position $[\mathrm{F}(1,13)=12.43$, $\mathrm{p}<.001, \eta^{2}=.489$ ], suggesting that targets were estimated to be different sizes based on where the object was located. Planned repeated contrasts revealed differences between target positions, and are shown in Figure 8. Targets in the ground position were judged as smaller than those in the below horizon position $(\mathrm{p}=.029)$. This finding is consistent with Experiment 1 where targets elevated in the absence of visible information to ground a target are judged to be both farther away and larger than targets with that information.

Additionally, size judgments to targets that were in the position above the visible horizon formed by the floor/wall boundary were significantly greater than those made to targets 
below the visible horizon $(\mathrm{p}=.002)$, demonstrating that participants judged above horizon targets to be larger than those below the horizon. Differences between the above horizon position and eye height position targets did not reach significance $(\mathrm{p}=.253)$.

The size judgments obtained in Experiment 2 were converted to indirect estimates of perceived distance using Equation (1). The value of $C$ determined for the non-visible stand condition of Experiment 1 was used, since this most closely matched the visual conditions of Experiment 2. Line-of-sight distances derived from Equation (1) were projected onto the floor so that distances were expressed in a manner consistent with Experiment 1. The values for these derived distances are displayed in Figure 9.

\section{Discussion}

Experiment 2 explored the size perception of targets located at different positions relative to a visible horizon. When targets were positioned above the ground surface but below a visible horizon (below horizon position), size judgments were overestimated compared to an on-ground target at the same distance. Given the relationship between size and distance judgments found in Experiment 1, we take this as evidence that targets were perceived as farther away when elevated. On other trials, targets were located above a visible horizon, visibly informing participants that the target could not be located on the ground plane. Such targets (both above horizon and eye height positions) were not only judged to be larger than targets on the ground plane, but also larger than off-ground targets below the visible horizon at the same actual distance. This suggests that participants perceived targets to be even farther when located above the visible horizon.

We were uncertain prior to the second experiment how objects would be perceived when they appeared above the visible horizon and so could not be located directly on the ground plane. Although further research is needed to determine precisely where targets are perceived, several of the previously discussed possibilities are inconsistent with our findings. One suggested outcome was that objects would be perceived at the location of the most plausible surface, in this case the back wall, unless there was information to suggest the contrary. In Experiment 2, distance judgments for targets above the visible horizon, as inferred from size judgments, averaged about $5.96 \mathrm{~m}$. This is well short of the actual distance to the back wall (9.83 meters). Although neither Experiment 1 nor Experiment 2 provided a direct indication of the perceived distance to the back wall, in the $4 \mathrm{~m}$ undetectable stand condition of Experiment 1, participants walked without hesitation to distances of $6 \mathrm{~m}$ and more, suggesting that the back wall was perceived as significantly farther. However, the possibility still exists that participants did perceive the targets as located on the location of the back wall, but were constrained by the narrow range of the target size. For instance, cognitive correction may have been used when making judgments for above horizon targets, understanding that it was unlikely that these targets were twice as large as those presented below the horizon. For a complete answer to this question, a larger range of target sizes would be an important follow-up.

Unlike Experiment 2, all of the targets in Experiment 1 appeared below the visible horizon formed by the floor-wall boundary of the room. In analyzing the results of Experiment 2, we used Equation 1 and the value of $C$ found in Experiment 1 to infer apparent distance for targets both below and above the visible floor-wall boundary. Additional studies are required to validate this inference for targets above the visible horizon and so not silhouetted against the floor. Similarly, as different groups of individuals were tested in Experiment 1 and Experiment 2, the inferred distance estimates calculated for Experiment 2 are somewhat limited. The different participant groups, as well as other differences in the experimental design and presented stimuli (i.e., target heights, target color), could also account for the slight differences in the magnitude of the underestimation of size seen between the 
detectable stand condition in Experiment 1 and the ground contact condition in Experiment 2.

The second possibility was that participants would treat the surface above the visible horizon as an extension of the ground plane. Support for this possibility would have been found with a trend of increased distance judgments as the target was elevated to a higher position, regardless of the targets position relative to the visible horizon. Our results suggest this is not an adequate explanation. Targets that were located at eye height did not differ from those located at a lower height. Lastly, we suggested the possibility that targets above the visible horizon would not be perceived along either the ground surface or the back wall surface. The findings of Experiment 2 are consistent with this third possibility. Although misperceived as farther away than objects that made contact with the floor, elevated objects located below a visible horizon were likely perceived as in contact with the ground plane. However, when objects were elevated above a visible horizon, targets were likely perceived as located off the ground plane. With no other visible information available, the perception of these targets fell in between the objects' veridical location and the back wall surface, although we presently cannot make claims about perception of the back wall surface itself. This account is further supported by a finding of increased within-subject variability in size judgments for positions above the visible horizon versus below: $\mathrm{SD}=.183, .177, .250$ and . 236 for ground, below horizon, above horizon and eye height, respectively, yielding a significant differences between below horizon and above horizon $(\mathrm{p}=.033)$ but not between on-ground and below-horizon positions $(\mathrm{p}=.863)$ or the two above-horizon conditions $(\mathrm{p}=$. 746). While our results of greater size judgments for targets that fell above versus below the horizon are consistent with the explanation that targets were perceived off the ground and in front of the back wall surface, there are likely other possible accounts as well. Future work is needed to further explain the mechanism leading to these differences.

\section{General Discussion}

It is well known that the ground plane provides a context that can aid in visual judgments of distance. This occurs both for objects resting on the ground surface and for objects above the ground that can be associated with a location on the ground surface through a visible support. Not previously known was whether this effect occurs under conditions of severely reduced contrast and acuity. We have shown that in fact nearly accurate distance judgments involving raised targets are possible, even when it is difficult or impossible to distinguish the supporting structure as anything more than an indistinct blob below the object of interest. We also showed that systematic errors occur in distance judgments to raised target locations when the support structures are not visible, and that the nature of these errors is different depending on whether the target appears below or above the visible horizon. These results have important implications for the design of architectural spaces accessible to those with profound low vision, since they highlight the necessity of visually tying potential obstacles to the ground so that distances to these obstacles can be accurately perceived.

In two experiments, participants viewed targets located off the ground plane in a simulated state of severely degraded acuity and contrast sensitivity. In Experiment 1, we showed that blind walking to previously viewed targets was performed accurately when visible information was available that highlighted the target's relationship with the ground plane. When the visible information associating the target and the ground plane was not available due to very low contrast between the support structure and the ground plane, participants overestimated the distance to target locations. This is consistent with the target being perceived as being approximately at the location on the flat ground plane that it occludes, as in the case with normal vision in the absence of visual information indicating a separation between target and ground. Further investigation is required to determine if information for 
the existence and shape of the ground plane under degraded visual conditions comes from visual information such as the walls, prior experience, or something else.

A second finding in Experiment 1 was that size judgments of viewed targets covaried with distance judgments in a manner consistent with the size-distance invariance hypothesis, suggesting that size judgments may be used as an indirect measure of distance perception in a state of simulated low vision. This is important for investigations of low vision distance perception, since the uncertainty associated with low vision can make response measures such as walking without vision to previously viewed targets impractical in constrained laboratory spaces. We exploited this effect in Experiment 2 to explore changes in perceived distance to targets as a function of where targets were located with respect to the visible horizon created by the boundary of the floor and wall surfaces. Inferred distance judgments for targets located off the ground plane, but below the visible horizon as defined by the boundary between the floor and the back wall, were larger than those for targets located at the same distance on the ground plane. For targets positioned above the visible horizon, we found that size judgments (and by implication distance judgments) were overestimated compared to targets that appeared below the visible horizon, but well short of being projected at the distance of the back wall.

Taken together, both experiments emphasize the importance of visible information that highlights an object's relationship with the ground surface in the recovery of absolute distance perception. Consistent with Gibson's ground theory, when viewers were presented with off-ground targets located below a visible horizon in the absence of cues to suggest their off-ground nature, their distance judgments were approximately consistent with an assumption that they were located on the ground plane.

As Gibson suggested, humans have acted and continue to act frequently with a terrestrial ground plane in the presence of gravity. It therefore makes sense that the visual system would assume an on-ground location of a target. However, it is also often the case that we are presented with off-ground objects in our environments. Our study suggests that if obstacles are elevated without clear visible information that connects the object to the ground plane, distances to the obstacles are overestimated. This means that a collision hazard exists, even when the obstacles themselves are visually detectable.

The hazards posed to navigation with off-ground obstacles undoubtedly increases for low vision individuals attempting to navigate with what visual abilities remain. It is important to understand which stimuli are relied upon under impaired viewing conditions in an attempt to maximize navigation potential for low vision populations and to inform orientation and mobility rehabilitation training. Although participants in our study were normally sighted individuals viewing targets through an induced state of profound low vision, general implications for design of accessible spaces are suggested. It is important to provide clear visible information grounding targets when they are located above the ground surface. As we found that targets will be perceived as farther away when this information is absent, this lack of information could present risks to navigation. When ground contact information is clearly visible, accurate distance perception is possible even under severely degraded viewing conditions. Future research should continue to build on our understanding of what cues are utilized by the low vision population to assist with navigation.

\section{Acknowledgments}

The authors thank Jonathan Bakdash for his assistance with data analysis, and Brandon Payne together with Benjamin Wheeler for their extensive assistance in data collection. This research was supported by NIH Grant 1 R01 EY017835-01. 


\section{References}

Durand A, Gould GM. A method of determining ocular dominance. Journal of the American Medical Association. 1910; 55(5):369-370.

Epstein W, Park J, Casey A. The current status of the size-distance hypotheses. Psychological Bulletin. 1961; 58(6):491-514. [PubMed: 13890453]

Fajen BR, Warren WH. Behavioral dynamics of steering, obstacle avoidance, and route selection. Journal of Experimental Psychology: Human Perception and Performance. 2003; 29(2):343-362. [PubMed: 12760620]

Fukusima SS, Loomis JM, Da Silva JA. Visual perception of egocentric distance as assessed by triangulation. Journal of Experimental Psychology-Human Perception and Performance. 1997; 23(1):86-100. [PubMed: 9090148]

Gilinsky AS. Perceived size and distance in visual space. Psychological Review. 1951; 58(6):460-482. [PubMed: 14900306]

Gibson, JJ. The perception of the visual world. Boston: Houghton-Mifflin; 1950.

Gogel WC. The sensing of retinal size. Vision Research. 1969; 9:1079-1094. [PubMed: 5350376]

Gogel, WC. The role of perceptual interrelations in figural synthesis. In: Dodwell, PC.; Caelli, T., editors. Figural synthesis. Hillsdale, NJ: Lawrence Erlbaum Associates; 1984. p. 31-82.

Gogel WC, Loomis JM, Newman NJ, Sharkey TJ. Agreement between indirect measures of perceived distance. Perception and Psychophysics. 1985; 37:17-27. [PubMed: 3991314]

Haber RN, Levin CA. The independence of size perception and distance perception. Perception \& Psychophysics. 2001; 63(7):1140-1152. [PubMed: 11766940]

Hocking, RR. Methods and applications of linear models: Regression and analysis of variance. 2. New York, NY: John Wiley; 2003.

Kilpatrick F, Ittelson W. The size-distance invariance hypothesis. Psychological Review. 1953; 60(4): 223-231. [PubMed: 13089000]

Kuyk T, Elliott JL, Biehl J, Fuhr PS. Environmental variables and mobility performance in adults with low vision. Journal of the American Optometric Association. 1996; 67(7):403-409. [PubMed: 8888866]

Long RG, Reiser JJ, Hill EW. Mobility in individuals with moderate visual impairments. Journal of Visual Impairment and Blindness. 1990; 84:111-118.

Loomis JM, Da Silva J, Fujita N, Fukusima SS. Visual space perception and visually directed action. Journal of Experimental Psychology: Human Perception and Performance. 1992; 1(4):906-921. [PubMed: 1431754]

Loomis, JM.; Philbeck, JW. Measuring perception with spatial updating and action. In: Klatzky, RL.; Behrmann, M.; MacWhinney, B., editors. Embodiment, ego-space, and action. Mahwah, NJ: Erlbaum; 2008.

Lovie-Kitchin JE, Soong GP, Hassan SE, Woods RL. Visual Field Size Criteria for Mobility Rehabilitation Referral. Journal of Optometry and Vision Science. 2010; 87(12):948-956.

Ludt R, Goodrich GL. Change in visual perception detection distances for low vision travelers as a result of dynamic visual assessment and training. Journal of Visual Impairment \& Blindness. 2002; 96(1):7-21.

Marron JA, Bailey IL. Visual factors and orientation-mobility performance. American Journal of Optometry \& Physiological Optics. 1982; 59(5):413-426. [PubMed: 7102800]

Meng JC, Sedgwick H. Distance perception mediated through nested contact relations among surfaces. Perception \& Psychophysics. 2001; 63(1):1-14. [PubMed: 11304007]

Meng JC, Sedgwick H. Distance perception across spatial discontinuities. Perception \& Psychophysics. 2002; 64(1):1-15. [PubMed: 11916293]

Mon-Williams M, Tresilian JR. The size-distance paradox is a cognitive phenomenon. Experimental Brain Research. 1999; 126:578-582.

Ono H, Muter P, Mitson L. Size-distance paradox with accommodative micropsia. Perception and Psychophyics. 1974; 15:301-307. 
Ooi TL, Wu B, He ZJ. Distance determination by the angular declination below the horizon. Nature. 2001; 141:197-200. [PubMed: 11700556]

Ozkan K, Braunstein ML. Background surface and horizon effects in the perception of relative size and distance. Visual Cognition. 2010; 18(2):229-254. [PubMed: 20814449]

Pelli, DG. The visual requirements of mobility. In: Woo, GC., editor. Low Vision: Principles and Applications. New York: Springer Verlag; 1987. p. 134-146.

Philbeck JW, Loomis JM. Comparison of two indicators of perceived egocentric distance under fullcue and reduced-cue conditions. Journal of Experimental Psychology-Human Perception and Performance. 1997; 23(1):72-85. [PubMed: 9090147]

Rand KM, Tarampi MR, Creem-Regehr SH, Thompson WB. The importance of the visible horizon for distance judgments under severely degraded vision. Perception. 2011; 40(2):143-154. [PubMed: 21650089]

Rieser JJ, Ashmead DH, Talor CR, Youngquist GA. Visual perception and the guidance of locomotion without vision to previously seen targets. Perception. 1990; 19(5):675-689. [PubMed: 2103000]

Sedgwick, HA. Environment-centered representation of spatial layout: Available visual information from texture and perspective. In: Beck, J.; Hope, B.; Rosenfeld, A., editors. Human and machine vision. New York: Academic Press; 1983. p. 425-458.

Tarampi MR, Creem-Regehr SH, Thompson WB. Intact spatial updating with severely degraded vision. Attention, Perception, \& Psychophysics. 2010; 72(1):23-26.

Thompson, WB.; Fleming, RW.; Creem-Regehr, SH.; Stefanucci, JK. Visual perception from a computer graphics perspective. Boca Raton: CRC Press; 2011.

Thomson JA. Is continuous visual monitoring necessary in visually guided locomotion. Journal of Experimental Psychology. 1983; 9(3):427-443. [PubMed: 6223981]

Turano KA, Broman AT, Bandeen-Roche K, Munoz B, Rubin GS, West S. SEE project team. Association of visual field loss and mobility performance in older adults. Journal of Optometry and Vision Science. 2004; 81(5):298-307.

\section{Appendix}

\section{Detectability study details}

A total of 6 individuals with normal to corrected-to-normal vision participated in a brief, 15minute study to assess the detectability of the black and gray stands used for Experiment 1. Each participant was first equipped with the noise-cancelling set-up used in Experiment 1 as well as the same blur goggles and hood. Participants were informed that they would be guided into the classroom and asked to make judgments about objects in the space in front of them. They were told that a white object would be present on each trial, and that their task would be to indicate whether or not the white object was on a stand. If the object was located on a stand, they were instructed to say 'yes'. If the object was not perceived to be located on a stand, they were instructed to say 'no'. Participants were then led into the classroom where they underwent 24 randomized trials: 6 trials with targets with a target on the gray stand, 6 trials with the target hanging from a cord with no stand present underneath, and 12 trials of a target on the black stand. On each trial, the location of the white sphere was unaltered. Participants were instructed to lift the hood and view the environment through the blur goggles. They were allowed to take as much time as needed to decide whether the object was located on a stand or not. After making their 'yes'/'no' response, participants lowered the hood and the new trial was set-up. Two target distances were tested, between subjects, to allow us to consider the variability of illumination conditions in the room. Three of the 6 participants viewed the target at a position 4 meters away, and the other 3 viewed targets located 2.7 meters from their location. Upon completion of the 24 trials, participants were led out of the classroom and thanked for their participation.

The results of the study confirmed that the gray stands were not detectable, and that the black stands were detectable. Every participant responded 'yes' to every trial where there 
was a black stand under the white object. Additionally, on each and every trial where a gray stand was located under the white object, participants responded 'no'. There were no exceptions across all 6 participants. When the target was positioned on a wire, with no stand underneath, participants almost always responded 'no'. There was only one participant who responded 'yes' to such a display, and this occurred only once. Taken together, we were confident that our gray and black stands were appropriate for Experiment 1 as they were undetectable and detectable, respectively. 

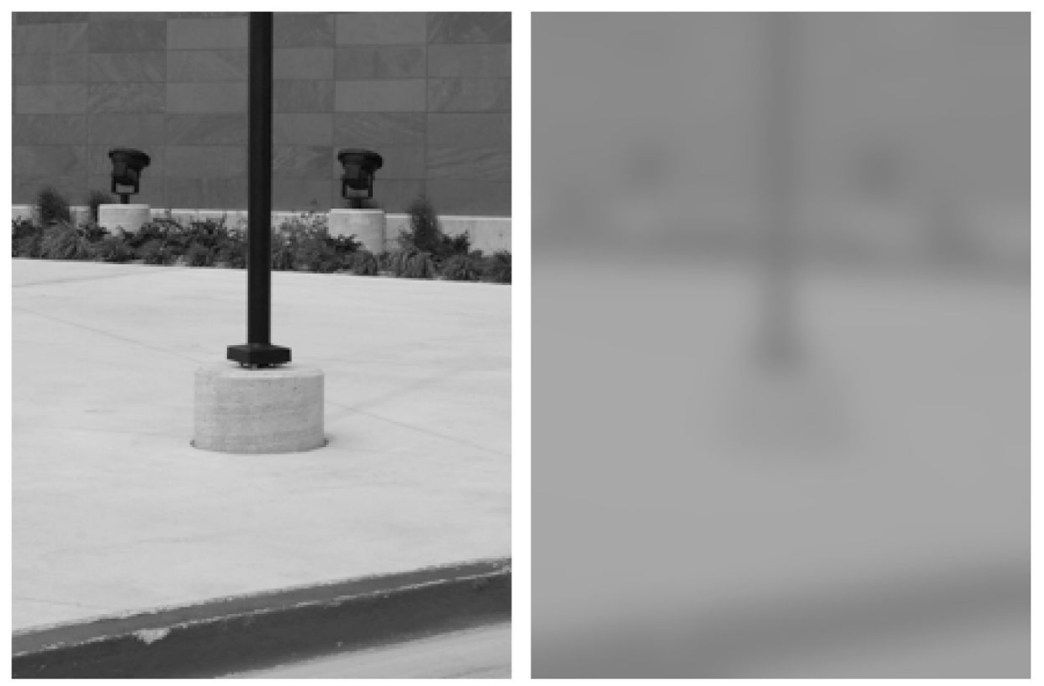

Figure 1.

Under conditions of extremely reduced acuity and contrast, it can be difficult to visually detect the concrete stand on which this light post rests. As a result, the post may be judged to be farther away than it actually is, thus representing a hazard to locomotion. 


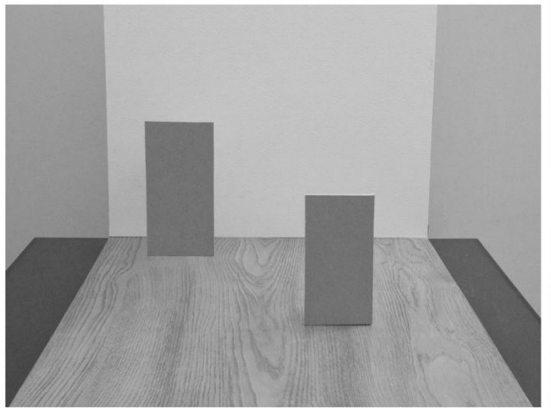

(a.)

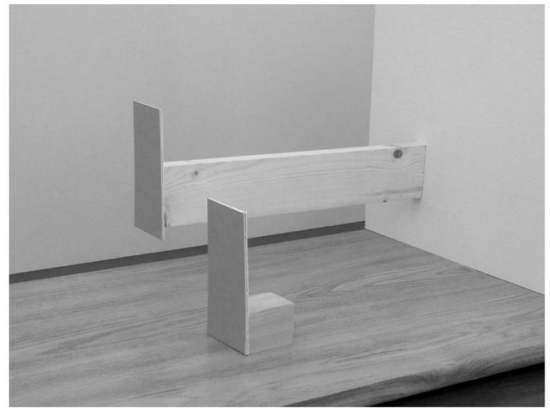

(b.)

Figure 2.

(a) Both rectangular objects appear in contact with a wooden support surface, with the left object seeming to be farther away than the right object, (b) The actual configuration, in which both rectangular objects are the same distance from the camera in (a), and the left object is not in contact with the support surface. (Motivated by a similar demonstration in Gibson, 1950). Image from Thompson, Fleming, Creem-Regehr, \& Stefanucci (2011). () 2011, CRC Press/An A. K. Peters Book, used by permission. 

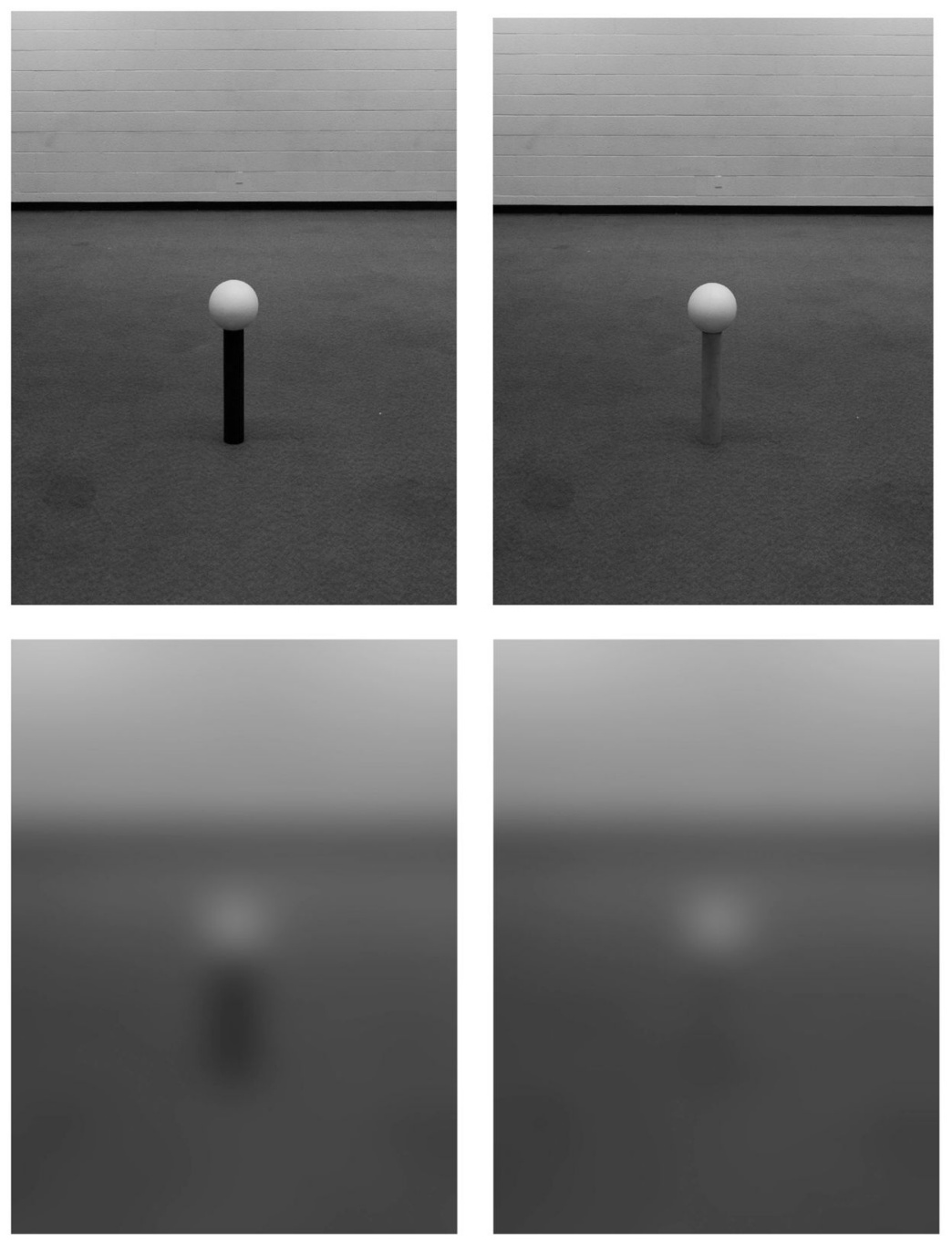

Figure 3.

Photographs of the detectable stand condition (left), and the undetectable stand condition (right) under normal viewing (above) and as participants viewed under degraded vision (below). Displayed degraded viewing conditions are stimulated for purposes of illustration, and do not correspond exactly to what was seen through the blur goggles. Participants did not view the stimuli under normal viewing conditions. 


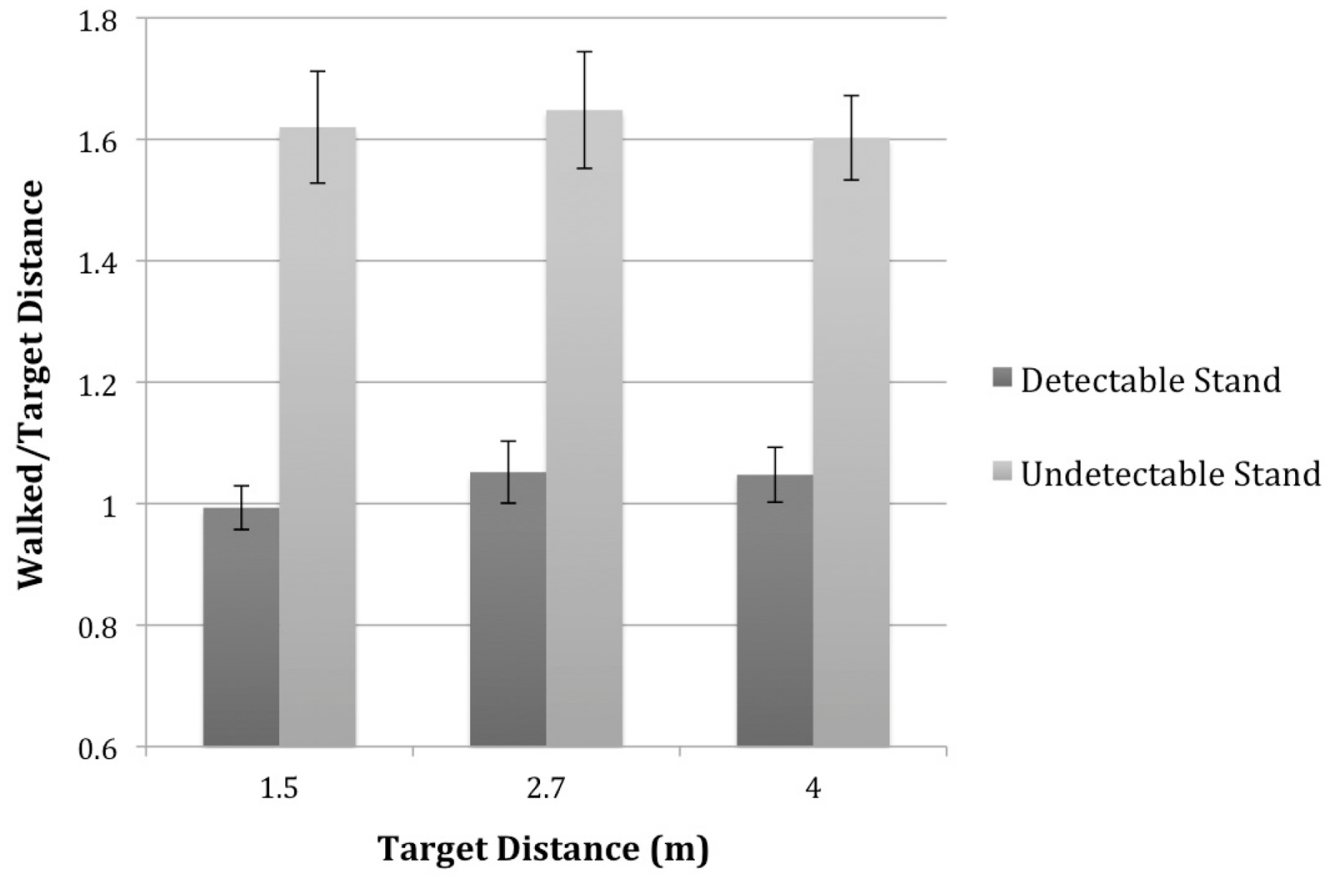

Figure 4.

Mean distance judgment ratios (walked/target distance) averaged over 6 trials of blind walking at each distance for each stand condition in Experiment 1. Error bars represent standard error. 


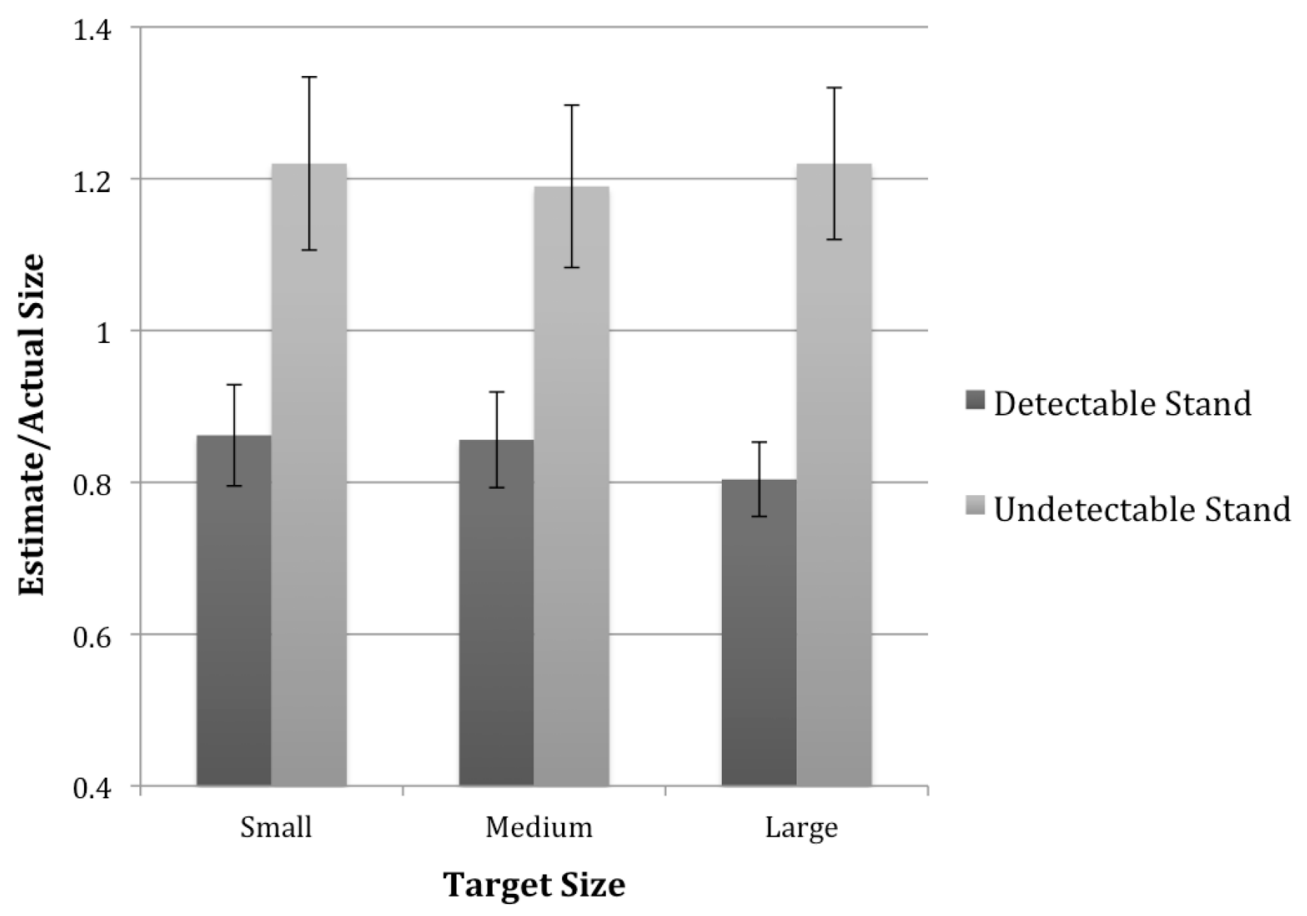

Figure 5.

Mean size judgment ratios (estimated/actual size) averaged over 6 trials of size judgments for each target size in Experiment 1. Error bars represent standard error. 

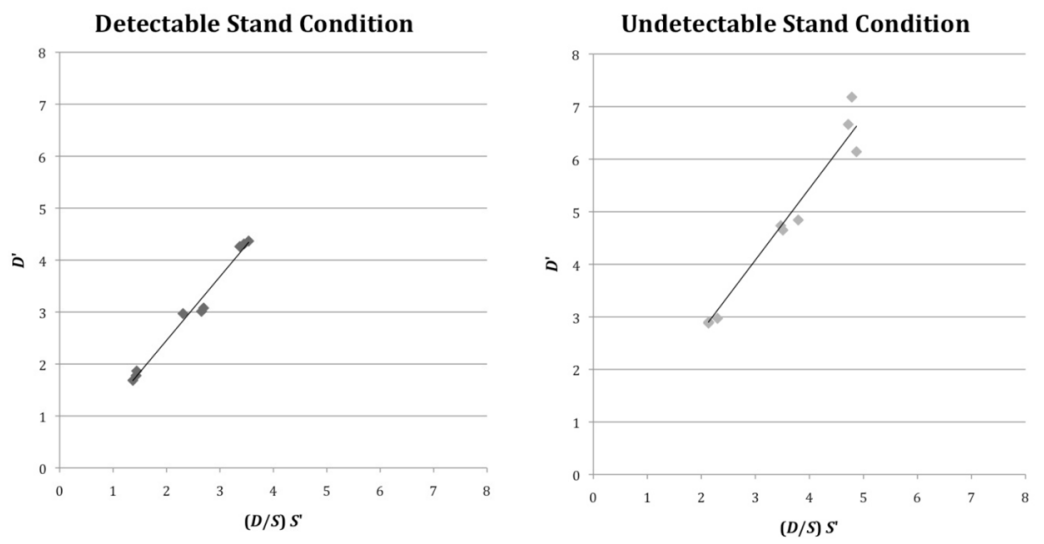

Figure 6.

Relationship between apparent size, $S^{\prime}$, and apparent distance, $D^{\prime}$, in Experiment 1. Each datapoint represents the average across all participants for each target size, at each target distance, for either the detectable or undetectable stands conditions. The horizontal axis shows $\left(\frac{D}{S}\right) S^{\prime}$, as in Equation 1. The lines show regression through the origin of the data values for each condition. All distances are with respect to the line-of-sight. 

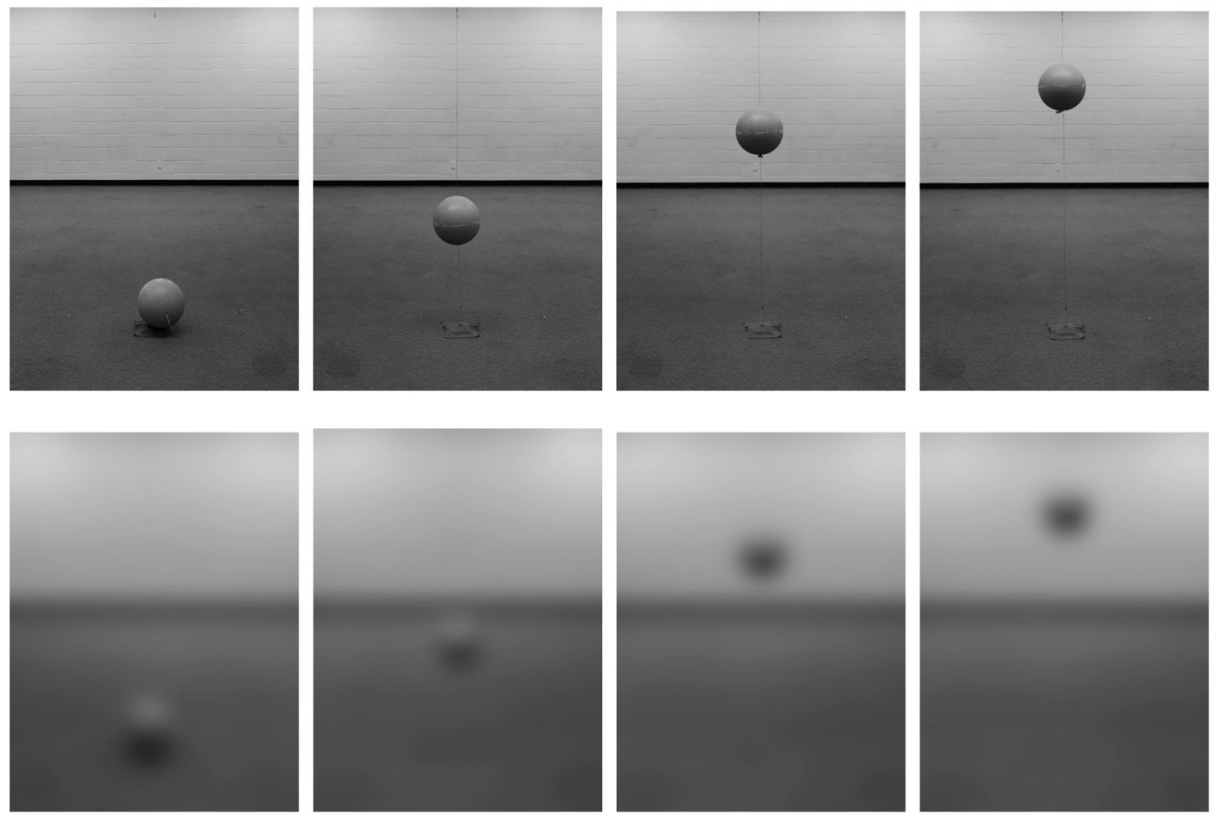

Figure 7.

Photographs of the hanging targets under normal viewing conditions (above) and as the participants viewed the targets under simulated profound low vision conditions (below). Displayed degraded viewing conditions are simulated for purposes of illustration, and do not correspond exactly to what was seen through the blur goggles. Targets are presented from left to right on ground, below horizon, above horizon, and eye height positions. The normal viewing condition was not presented to the participants. 


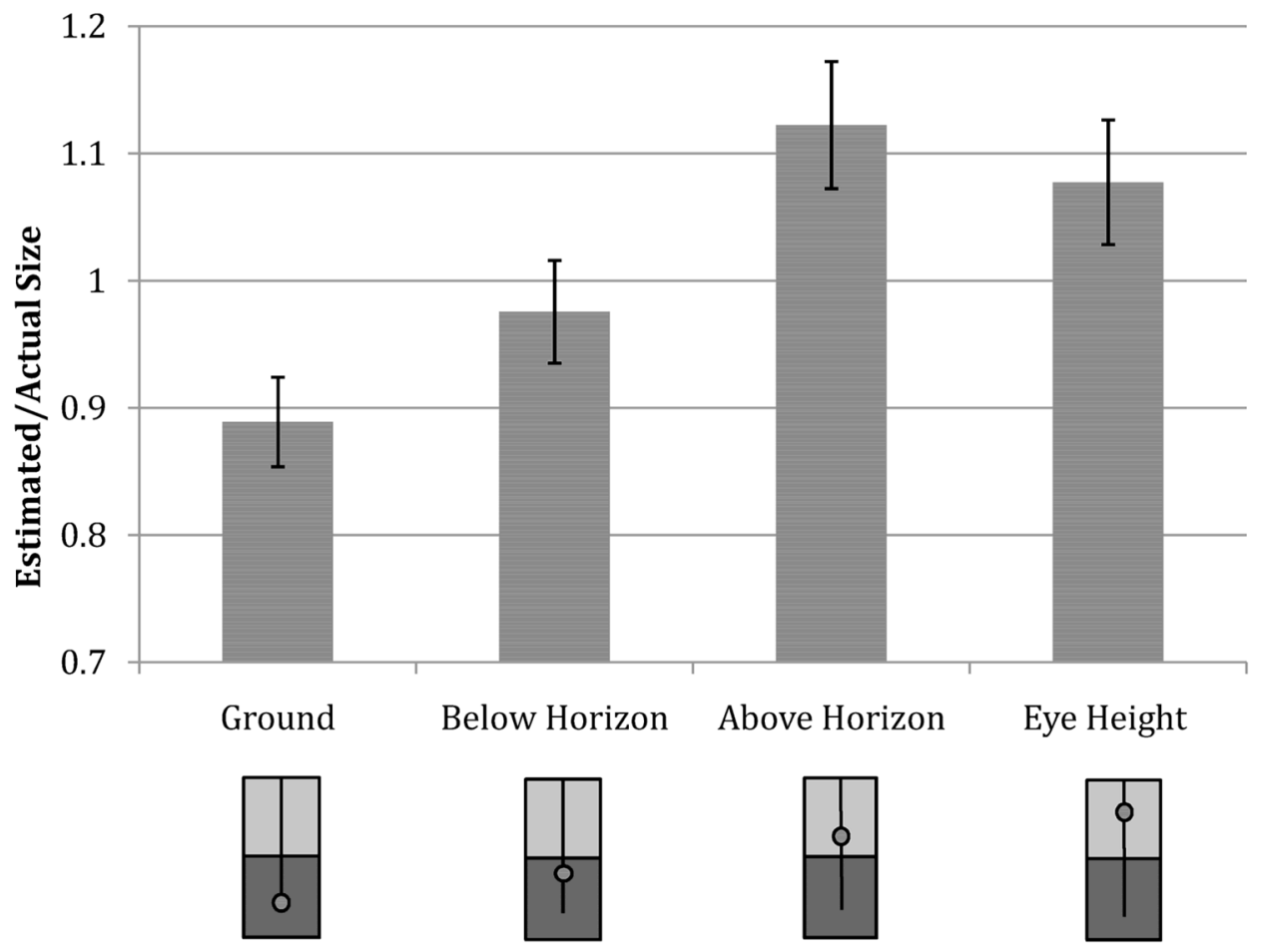

Target Position

Figure 8.

Ratio of estimated size judgments over actual target size for 24 trials of size judgments for Experiment 2. Error bars indicate standard error. 


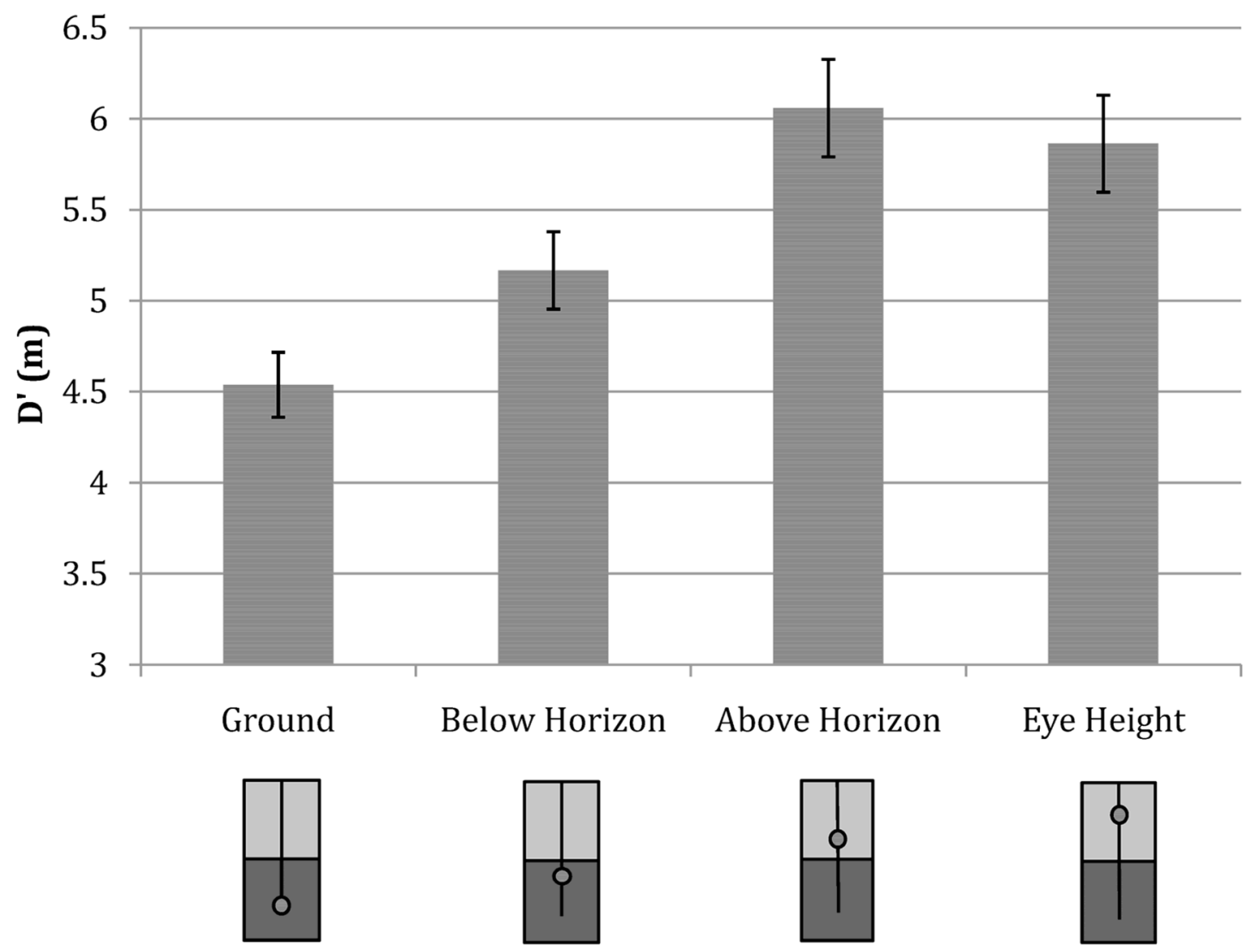

Target Position

Figure 9.

Average apparent distance value, $D^{\prime}$, based on Equation (1) with the value of $C$ as determined for the non-visible stand condition in Experiment 1, with distances projected along the floor. 